\section{Transituational control of avoidance responding by Pavlovian $\mathrm{CSs}^{1}$}

OTELLO DESIDERATO, Connecticut College, New London, Conn. 06320

After dogs learned Sidman avoidance in a shuttlebox, they received differential Pavlovian conditioning in a different room, strapped to a harness. Shock was paired with one tonal stimulus (CS+) but not with the second (CS-). A control group received both tones but no shock. Pavlovian CSs were later introduced while Ss were performing the avoidance response in the shuttlebox. While CS+ increased avoidance responding, neither $C S-$ nor tones in the control group significantly inhibited avoidance behavior.

Recent research (Desiderato, 1969; Rescorla \& LoLordo, 1965) has shown that a stimulus that is explicitly paired with shock (CS+) will later enhance response rate when superimposed on a Sidman shock-avoidance schedule; conversely, a stimulus that has been explicitly nonpaired with shock (CS-) will depress avoidance responding. These results have been interpreted as being consistent with Pavlovian concepts of excitation and inhibition and with two-process theory (Rescorla \& Solomon, 1967).

In most of the studies demonstrating such control of instrumental behavior by Pavlovian CSs, the same (or similar) apparatus has been used for both the classical conditioning and the shock-avoidance phases. In one of the few studies that attempted to demonstrate transituational transfer (from Pavlovian harness to shuttlebox), Leaf (1964) produced evidence of control by $\mathrm{CS}+$ but not by $\mathrm{CS}-$, since the signalized avoidance procedure he employed provided no baseline against which to assess inhibitory effects.

The present study was undertaken to study transituational transfer of excitation and inhibition, from Pavlovian harness to shuttlebox, by observing the effect of introducing Pavlovian CSst and CSs-- on the rate of Sidman avoidance responding. METHOD

The Ss were 20 mongrel male dogs, obtained from a local supplier. They were housed in individual cages and maintained

Fig. 1. Mean number of avoidance responses during $\mathrm{CS}+$ and $\mathrm{CS}-$ in Group $\mathbf{E}$, during tones in Group $\mathrm{C}$, and during 5 -sec periods before stimulus presentations in both groups (baseline). noise level. on ad lib food and water throughout the experiment. Three Ss were discarded because of illness, two for failure to learn the avoidance response, and one for failure to respond on the test day. The remaining 14 dogs were evenly divided between experimental and control groups.

A Pavlovian harness, described in detail elsewhere (Black, 1958), consisted of a rubberized hammock through which the S's legs hung through four holes. The dog's body and legs were secured by straps. Shock of $4.5 \cdot \mathrm{mA}$ intensity could be delivered to the footpads of the S's hind feet through brass plates coated with electrode paste. The entire unit was located inside a white, sound-attenuated cubicle, into which tonal stimuli could be introduced about $15 \mathrm{~dB}$ above the intensity level of a masking white noise of $70 \mathrm{~dB}$ SPL. Speakers within each compartment were used to deliver tonal stimuli about $15 \mathrm{~dB}$ above the general

On the first 3 days, each $S$ was trained to jump the barrier in the shuttlebox to avoid shock. Using an unsignaled Sidman avoidance schedule, every jump postponed shock for $30 \mathrm{sec}$. If $\mathrm{S}$ failed to jump, shock was delivered to the grid every $10 \mathrm{sec}$. All shocks were $.25 \mathrm{sec}$ in duration. The height of the barrier was 9 in. on the first day, 12 in. on the second, and 15 in. thereafter. On the fourth day, each $\mathrm{S}$ was secured to the harness for the first Pavlovian conditioning session, which consisted of 30 trials. For four $S s$ in the experimental group

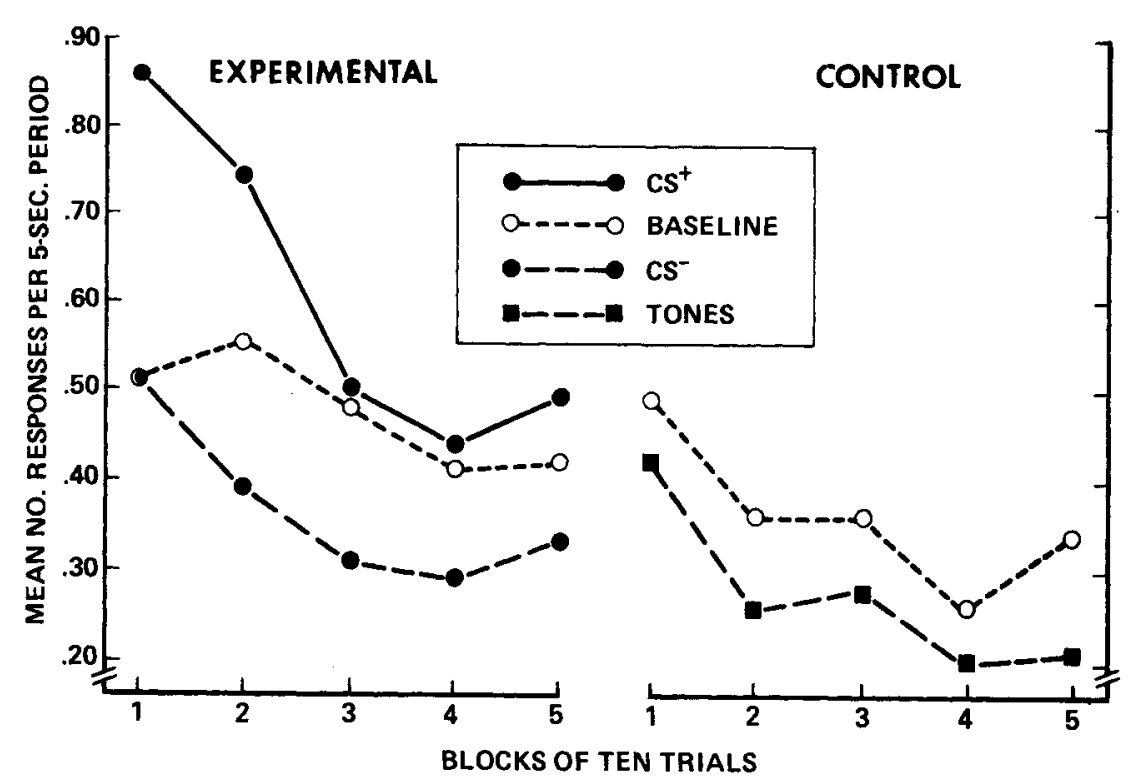

(Group E), CS+ was a 5-sec, $1,200-\mathrm{Hz}$ tone, whose termination was always followed, after 2,5 , or $8 \mathrm{sec}$, by $5 \mathrm{sec}$ of continuous shock. The CS-, a 5-sec, 400-Hz tone, was never followed by shock. For the remaining three Ss in Group $E$, the tones were reversed. The seven $\mathrm{Ss}$ in the control group (Group C) received identical treatment, but shock was never used. All Pavlovian sessions consisted of $15 \mathrm{CS}+$ and $15 \mathrm{CS}$ - trials, presented in random order. ${ }^{2}$ The intertrial interval was 30,60 , or $90 \mathrm{sec}$, the duration varying randomly within sets of three. Pavlovian sessions were given every other day, alternating with avoidance sessions, until a sequence of six Pavlovian and eight avoidance sessions was completed. The duration of all sessions was $1 \mathrm{~h}$ per day.

On the 15th day, a single test session was given. While the $S$ was performing the avoidance response, CSt and CS- were presented in random order, each for 5-sec durations. Each stimulus was presented 50 times, the intertrial interval varying randomly within sets of three values: 20 , 35 , and $50 \mathrm{sec}$. No shock was given. RESULTS

Five $S s$ in Group $E$ and four in Group $C$ learned the avoidance response by the end of the first session. All Ss in both Groups E and $\mathrm{C}$ were responding at a stable rate by the last avoidance session, averaging 8.4 and 6.4 jumps per minute, respectively. During the test session, the response rate for Groups $E$ and $C$ was 6.81 and 4.81 , respectively, a substantial, but not significant, difference according to the Mann-Whitney Test $(\mathrm{U}=13, \mathrm{p}<.17$, two-tailed test).

The number of avoidance responses occurring during the 50 presentations of CSt and of CS- was recorded for each S. 
Mean response rates for both groups, over blocks of 10 trials, are shown in Fig. 1. This figure also shows baseline response rates, computed by averaging response rates during the 5 -sec periods immediately preceding CSt and $\mathrm{CS}-$ in the case of Group $\mathrm{E}$ and immediately preceding the same two tones in Group C.

The left panel of Fig. 1 indicates that, during the early test trials, the presentation of CS+ increased the emission of avoidance responses in Group $\mathrm{E}$ considerably above its baseline rate. This increase is significant only for the first block of 10 trials, however (Wilcoxon $\mathrm{T}=1, \mathrm{p}<.05$ ). It is also apparent that the response rate in the presence of $\mathrm{CS}-$ was considerably lower than the baseline rate during Test Blocks 2 to 4 , but for no test block did the value of $\mathrm{T}$ attain the $5 \%$ level of significance for two-tailed tests. The same conclusion applies to the differences between baseline responding and response rate in the presence of the tones in Group C, although the mean response level was lower during the tones for each of the five test blocks. To compare the amount of below-baseline responding produced in the presence of $\mathrm{CS}$ - in Group E with that occurring in the presence of the tones in Group C, for each $\mathrm{S}$ the difference between baseline rate and rate in the presence of CS- (or tones) was computed across all test trials. These differences, compared by means of the Mann-Whitney U Test, were no greater for Group E than they were for Group C $(U=17)$. In Fig. 2, the difference between each group's mean baseline rate and its mean level of avoidance responding in the presence of $\mathrm{CS}+$ and $\mathrm{CS}-$ (Group E) or in the presence of tones (Group C) is shown, plotted against trial blocks. Mann-Whitney U Tests revealed that for none of the five test blocks was there a significant difference between tones and $\mathrm{CS}-$ in amount of deviation from baseline.

\section{DISCUSSION}

The results confirm the findings of Rescorla \& LoLordo (1965) that a stimulus noncontingently paired with shock will enhance the rate of nondiscriminated avoidance responding. As in their Experiment 2, which used a fixed CS-US interval rather than the variable interval employed in the present study, the main excitatory effect occurred primarily during the first 10 test trials. The rapid dissipation of the effect of CS+ contrasts with the protracted excitatory effect which Rescorla and LoLordo observed in their Experiment 1, in which the nonreinforced Pavlovian trials consisted of CS+ followed by CS-. Apparently, the effects of conditioned inhibition are more resistant to extinction than are those of differential inhibition.

A major conclusion of the present study is that excitatory control by a Pavlovian CS+ will transfer from the original classical conditioning situation, in which the stimulus acquires its effectiveness, to a different setting in which the $S$ is performing an aversively motivated instrumental response. In addition to providing evidence of transituational transfer of excitatory effects for dogs, the present results extend the generality of a recent finding by Grossen \& Bolles (1968). These investigators reported that rats trained on a Sidman avoidance schedule to make a hurdle-jumping response in a shuttlebox responded with shorter latencies when a CS+ was introduced than when the stimulus had either (1) occurred randomly in time with shock in a prior session conducted in a separate conditioning apparatus or (2) had never been experienced prior to the test session.

The current findings, however, reveal that a $\mathrm{CS}-$ produces no greater inhibition of the avoidance response than do stimuli that had been presented in "Pavlovian conditioning" sessions in which shock had never been present. While this result supports Rescorla and LoLordo's conclusion that mere experience with a stimulus in the absence of shock is not sufficient to make the stimulus inhibitory, it does not confirm their finding that stimuli occurring without shock, against a background in which shock does in fact occur, acquire the capacity to inhibit avoidance responding. Nor do these results confirm Grossen \& Bolles's (1968) report for rats of retarded hurdle-jump latencies to a stimulus that had always occurred separated in time from shock.

The failure to find transfer of inhibitory effects of $\mathrm{CS}$ - in Group E may have resulted from too great a change in situational (i.e., room and apparatus) cues in passing from the Pavlovian to the instrumental avoidance setting. This explanation, however, presumes that (1) the inhibitory control acquired by $\mathrm{CS}$ during Pavlovian conditioning was weaker than the excitatory effect acquired by CS+, or that (2) the generalization decrement for $\mathrm{CS}_{-}$- is greater than that for CS+, or (3) both. The present study does not discriminate between these various possibilities.

\section{REFERENCES}

BLACK, A. H. The extinction of avoidance responses under curare. Journal of Comparative \& Physiological Psychology, 1958, 51, 519-525.

DESIDERATO, 0 . The generalization of excitation and inhibition in the control of avoidance responding by Pavlovian CSs in dogs. Journal of Comparative \& Physiological Psychology, 1969, 68, 611-616.

GROSSEN, N. E., \& BOLLES, R. C. Effects of a classical conditioned 'fear signal' and 'safety signal' on nondiscriminated avoidance behavior. Psychonomic Science, 1968, 11, 321-322.

LEAF, R. C. Avoidance response evocation as a function of prior discriminative fear conditioning under curare. Journal of Comparative \& Physiological Psychology, 1964, 58, 446-449.

RESCORLA, R. A., \& LoLORDO, V. M. Inhibition of avoidance behavior. Journal of Comparative \& Physiological Psychology, $1965,59,406-412$.

RESCORLA, R. A., \& SOLOMON, R. L. Two-process learning theory: Relationships between Pavlovian conditioning and instrumental learning. Psychological Review, $1967,74,151-182$.

SOLOMON, R. L., \& WYNNE, L. C. Traumatic avoidance learning: Acquisition in normal dogs. Psychological Monographs, 1953, 67(4, Whole No. 354).

\section{NOTES}

1. This research was conducted at the University of Pennsylvania, where the author was

Fig. 2. Difference in mean number of responses during $5-\mathrm{sec}$ periods during and preceding presentations of $\mathrm{CS}+$ and $\mathrm{CS}-$ (Group E) and tones (Group C). 
an $\mathrm{NSI}^{-}$Science laculty Fellow. Support for the rescarch came from USPHS Grant MH-04202 and NSF: Grant GB-2428, both to Richard $L$. Solomon. I am indebted to Richard L. Solomon for his advice and criticism and to Lucille $H$.
Turner and Donald Hanson for their technical assistance.

2. On the final Pavlovian session, one $S$ in Group E received only 12 trials $(6 \mathrm{CS}+$ and 6 CS J, due to apparatus failure.

\section{Negative incentive contrast with sucrose ${ }^{1}$}

\section{IAWRENCE WEINSTEIN, ${ }^{2}$ University of Maine, Portland, Maine 04103}

A decrease in the concentration of a sucrose solution resulted in negative incentive contrast effects. This finding disagrees with many studies in the literature.

When a decrease in incentive size results in a decrement in performance significantly below a baseline defined by Ss trained only at the postshift magnitude, the result is referred to as negative incentive contrast effect. While negative incentive contrast effect has been well documented (e.g., Black, 1968), many investigators have failed to obtain the phenomenon using sucrose as incentive (e.g., Rosen, 1966). Rosen (1966) and Dunham \& Kilps (1967) decreased the concentration of a sucrose solution and failed to obtain negative contrast effects. This may be because they selected preshift concentrations not conducive to the production of negative incentive contrast effects. DiLollo \& Beez (1966) have shown that the magnitude of negative contrast effects is a positive function of the amount of reward reduction where larger and smaller rewards are defined in terms of whether they produce higher or lower levels of performance, respectively. Guttman (1953) reported maximum and minimum bar pressing to a $16 \%$ and $4 \%$ sucrose solution, respectively, while $8 \%$ and $32 \%$ solutions produced intermediate levels of performance. Rosen (1966) reduced the concentration of a sucrose solution from $20 \%$ to $3 \%$ in a runway.

Dunham \& Kilps (1967) reduced the concentration of a sucrose solution from $32 \%$ to $11.3 \%$ following training in an operant conditioning box. Perhaps larger reductions in amount of reward would produce negative incentive contrast effects. Guttman's (1953) data suggest that a shift in concentration from $16 \%$ to $4 \%$ would constitute a larger change in incentive size than would the procedures used by Rosen (1966) and Dunham \& Kilps (1967) and would, therefore, be more likely to result in negative incentive contrast effects. The present experiment examined a shift in the concentration of a sucrose solution from $16 \%$ to $4 \%$.

\section{SUBJECTS}

The Ss were 20 experimentally naive hooded rats, 75-105 days old at the start of the experiment and housed in individual cages.

\section{APPARATUS}

The $12 \times 10 \times 10$ in. conditioning chamber had plywood side walls, Plexiglas top, back, and door, and a grid floor.

A rectangular hole, $1 / 2 \times 2$ in., was cut in the side of the cage 5 in. above the floor so that the bar could be inserted $1 / 2$ in. into the cage. Three inches below and 2 in. to the side of the bar, a circular hole, 1 in. in diam, was cut in the wall. Through this opening, $S$ had access to the liquid magazine.

The liquid used was presented to $S$ by a dipper-type magazine driven by a motor. A metal drinking tube, attached to a $3-\mathrm{ft}$ piece of plastic tubing that connected to a plastic cylinder graduated in $\mathrm{cc}$, was mounted $1 \mathrm{in}$. outside the circular hole in the wall so that whenever $S$ pressed the bar the tip of the tube was presented through the hole for $2 \mathrm{sec}$ so that $\mathrm{S}$ could lick the tube by extending his tongue through the hole. During the 2-sec dipper presentation, bar presses were recorded and did not operate the dipper.

Events were programmed and recorded automatically by a BRS solid-state system located in the experimental room. Bar presses were recorded by means of a counter.

\section{PROCEDURE}

Ten Ss were assigned randomly to each of two groups. The control group (C) received $4 \%$ sucrose throughout the experiment and the other group (S) was shifted from $16 \%$ to $4 \%$ on the 10 th session, after bar-press asymptote was reached.

Following several days of adaptation to a food-deprivation schedule of pellets and water for $1 \mathrm{~h}$ at the end of the experimental time, daily, Ss were trained to press the bar for continuous reinforcement (with each depression the bar operated the magazine). Each session lasted for 40 reinforcements (dipper presentation). This regime was continued until performance appeared to be stable, requiring nine sessions (preshift). Concentration was then changed (postshift) and all Ss were continued through the 13th session. Throughout the study, Ss had access to dry pellets and water for $1 \mathrm{~h}$ following the experimental period.

\section{RESULTS}

Average bar presses per minute (number of bar presses in the session/session duration) were examined in the analysis of the results.

From Fig. 1, it seems clear that, from Sessions 1-9, 16\% sucrose produced a higher rate of bar pressing than did $4 \%$ sucrose. The mean number of bar presses per minute from Sessions 1-9 differed significantly between the groups by analysis of variance $[F(1,18)=9.43$, $\mathrm{p}<.01]$.

Figure 1 indicates that on Session 10, the $S$ group suddenly reduced their bar-press rate below that of the control group (negative contrast effect). A 4 by 2 analysis of variance of the mean number of bar presses per minute over the four postshift sessions and the two concentrations revealed a significant concentration effect $[F(1,18)=4.72$, $\mathrm{p}<.05]$ and a statistically reliable interaction $[F(3,18)=3.19, \mathrm{p}<.05]$.

The mean number of bar presses per minute from Sessions 10-11 differed significantly between the $\mathrm{S}$ and $\mathrm{C}$ Ss (negative incentive contrast effect) by analysis of variance $[F(1,18)=4.53$, $p<.05]$

The finding that a $16 \%$ sucrose solution produces a significantly higher level of performance than does a $4 \%$ solution agrees with Guttman (1953) and indicates that $S$ can discriminate the difference in concentration.

The demonstration of negative incentive contrast effects with a shift in concentration of a sucrose solution disagrees with many studies in the literature (e.g., Dunham \& Kilps, 1967; Guttman, 1953; Rosen, 1966). The discrepant findings may be due to the different (1) deprivation conditions and/or (2) preshift concentrations selected by the present investigation and the three studies previously cited.

In the present investigation, Ss were deprived of both food and water, while Dunham and Kilps, Guttman, and Rosen deprived their Ss only of food. Quite possibly the present more severe 